Palabras clave/ Edificios educacionales, energía solar, células fotovoltaicas, optimización.

$\Delta$ Keywords/ Educational buildings, solar energy, photovoltaic cells, optimization.

$\Delta$ Recepción/ 1 julio 2017

$\Delta$ Aceptación/ 4 diciembre 2017

\section{Forma arquitectónica e integración fotovoltaica en edificios educacionales de Concepción, Chile ${ }^{1}$}

\section{Architectural Shape and Photovoltaic Integration in Educational Buildings in Concepcion, Chile ${ }^{1}$}

\section{Daniel Sosa-Ibarra}

Arquitecto, Universidad de la República

Uruguay.

Magíster en Hábitat Sustentable y Eficiencia Energética, Universidad del Bío-Bío, Chile. Académico, Departamento de Clima y Confort en la Arquitectura, Universidad de la República, Montevideo, Uruguay. danielsosaibarra@gmail.com

\section{Rodrigo García-Alvarado}

Arquitecto, Pontificia Universidad Católica de Chile, Chile

Doctor en Arquitectura, Universidad Politécnica de Cataluña, España.

Académico, Departamento de Diseño y Teoría de la Arquitectura, Universidad del Bío-Bío, Concepción, Chile. rgarcia@ubiobio.cl

RESUMEN/ Los edificios educacionales constituyen servicios de gran relevancia social, localizándose dispersamente en la ciudad pero en volúmenes extensos. Además, presentan consumos energéticos crecientes y un limitado desempeño ambiental, lo que puede mejorarse mediante sistemas fotovoltaicos integrados en cubierta, debidamente orientados para maximizar sus capacidades. Este trabajo presenta una evaluación de las morfologías educacionales, para determinar la capacidad solar activa en cubierta y sugerir formas con mejores desempeños a partir de estudios de establecimientos en Concepción, Chile. Un análisis multifactorial permite descifrar la posición óptima de paneles fotovoltaicos, comprobando variaciones en la generación de energía. Luego, se determina el aporte energético solar suficiente para cubrir el consumo de 4 plantas en ciclo básico-medio y 3 en superior, y con remanentes que pueden mejorar las condiciones de confort, compartirse localmente o volcarse a la red. La información recopilada se implementa en una definición paramétrica que puede asistir decisiones tempranas de diseño de nuevos edificios educacionales. ABSTRACT/ Educational buildings are services with a strong social relevance, scattered around the city but with extensive dimensions. In addition, they have increasing energy intensity and limited environmental performance, which could be improved with building-integrated photovoltaic systems, duly oriented to maximize their capacities. This work introduces an evaluation of educational morphologies to determine the building's active sun capacity and propose improved performance shapes based on the study of facilities in Concepción, Chile. A multifactorial analysis allows identifying the optimal position of photovoltaic panels, verifying variations in energy generation. Then, the solar energy required to meet the consumption of four stories in the elementary-middle school cycle and three in high-school is determined, with surpluses that can enhance comfort, be shared locally or overturned to the grid. The information collected was implemented in a parametric definition to inform early design decision-making of new educational buildings.

INTRODUCCIÓN.

mundial vive actualmente en ciudades, siendo Chile uno de los países más urbanos del mundo, con el $90 \%$ de la población en áreas urbanas (Naciones Unidas 2014) y un consumo energético creciente acoplado al desarrollo socio-económico (Ministerio de Energía, Gobierno de Chile 2014a). En este escenario, la energía solar ofrece una de las mejores posibilidades de suministro renovable en zonas urbanas, debido a sus posibilidades de interconexión (Lund 2012). Esta lógica, analizada para la ciudad de
Concepción (García, Wegertseder, Baeriswyl y Trebilcock 2014), podría hacerse extensiva a las infraestructuras educacionales, considerando sus características de dispersión urbana y extensión volumétrica, dando así respuesta al creciente consumo energético y limitado desempeño ambiental mediante la incorporación de sistemas fotovoltaicos en cubierta para generación de energía eléctrica, a partir de estrategias de diseño que maximicen el potencial solar. Mientras que la enunciación tradicional de condiciones de forma para el mejor desempeño energético de las edificaciones se centraba en el equilibrio entre las tensiones térmicas de interior y exterior (Depecker, Menezo, Virgone y Lepers 2001; García y González 2014; Olgyay 1962), la declaración contemporánea puede vincularse a una optimización del potencial solar. Esta condición se justifica aún más al considerar el bajo rendimiento de las tecnologías fotovoltaicas -amorfo 6-8\%, multi-cristalino 13-16\%, mono-cristalino 15-18\%- (Tripathy, Sadhu y Panda 2016), y los elevados costos de inversión inicial. 
Algunos trabajos han investigado las incidencias de las variaciones formales del diseño de cubierta sobre el potencial solar de viviendas unifamiliares de latitudes medias (Hachem, Athienitis y Fazio 2011)

En el contexto regional, el estudio del potencial de captación solar asociado a las características formales de cubierta en viviendas unifamiliares, ha probado que puede suplir el promedio de consumo eléctrico anual de una vivienda (Zalamea y García 2014). Estas exploraciones formales adquieren una nueva dimensión con herramientas de diseño paramétrico que, combinadas con plugins de análisis ambiental, permiten que los proyectistas realicen una integración temprana de aspectos energéticos en el desarrollo de la forma arquitectónica, transformando el diseño en una respuesta basada en análisis de desempeño (Anton y Tănase 2016) e identificando la solución más eficiente para resolver un problema mediante algoritmos genéticos (AG) (Salcedo 2012). Algunos estudios combinan AG con simulación energética para desarrollar herramientas que permitan optimizar la forma de las edificaciones, minimizando el uso de energía en tipologías residenciales (Tuhus-Dubrow y Krarti 2010). En el contexto local, otras experiencias utilizan simulación dinámica y AG para generar un diseño paramétrico de mansarda con integración de tecnologías solares como extensión de la vivienda unifamiliar y generando una solución adaptativa variable según la orientación (Troncoso, García, Nope y Arellano 2015).

Estas experiencias muestran, desde distintos enfoques, relaciones entre forma y potencial solar, pero estas condiciones no han sido revisadas para establecimientos educacionales. Este trabajo realiza un análisis de los establecimientos educacionales de la comuna de Concepción, Chile, generando, en base a sus características formales y energéticas, una definición de diseño paramétrico que tiene por objetivo determinar la capacidad solar de cubierta en función de su volumetría, sugiriendo formas optimizadas con mejores desempeños.

\section{CARACTERÍSTICAS DE ESTABLECIMIENTOS EDUCACIONALES EN LA COMUNA DE CONCEPCIÓN. Catastro de establecimientos educacionales.}

La comuna de Concepción cuenta con una población de 216.061 habitantes (Instituto Nacional de Estadística de Chile 2002) y forma parte del área metropolitana del Gran Concepción, localizada en la latitud $36^{\circ} 49^{\prime} 37^{\prime \prime} S$ y longitud 7302'59'O, en la zona centro-sur de Chile, Región del BíoBío. Posee un clima templado húmedo, influenciado por la proximidad al océano que modera las oscilaciones térmicas diarias y anuales, una temperatura media de $13^{\circ} \mathrm{C}$ (World Meteorological Organization s/f) y una radiación solar global horizontal de 1642,5 kWh/m²-año (Ministerio de Energía Gobierno de Chile 2014b).

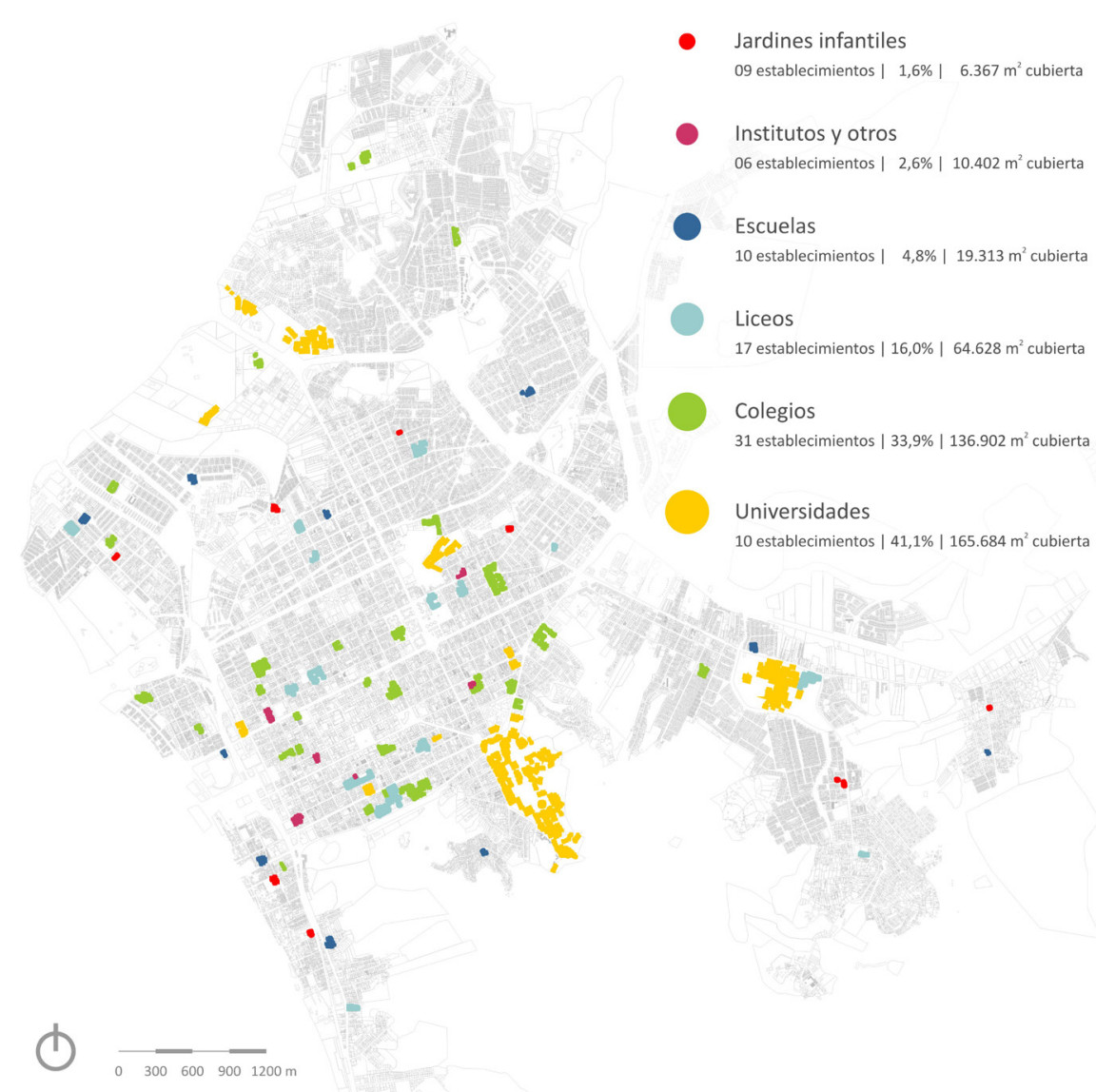

Figura 1. Establecimientos educacionales en la comuna de Concepción (fuente: Elaboración propia).

La región cuenta con gran cantidad de establecimientos educacionales, siendo la segunda del país en superficie total de infraestructura escolar, pero presentando importantes problemas de materialidad y sistemas precarios en las instalaciones (Ministerio de Educación, Gobierno de Chile s/f)

La revisión del parque educacional existente en la comuna permite reconocer las características representativas de los establecimientos. Los antecedentes generales de infraestructura (García et al. 2014) se complementan con registros de servicios locales, identificando 83 establecimientos correspondientes a un $82,7 \%$ de los servicios.

La figura 1 muestra el relevamiento de establecimientos educacionales en la comuna de Concepción y la dispersión 


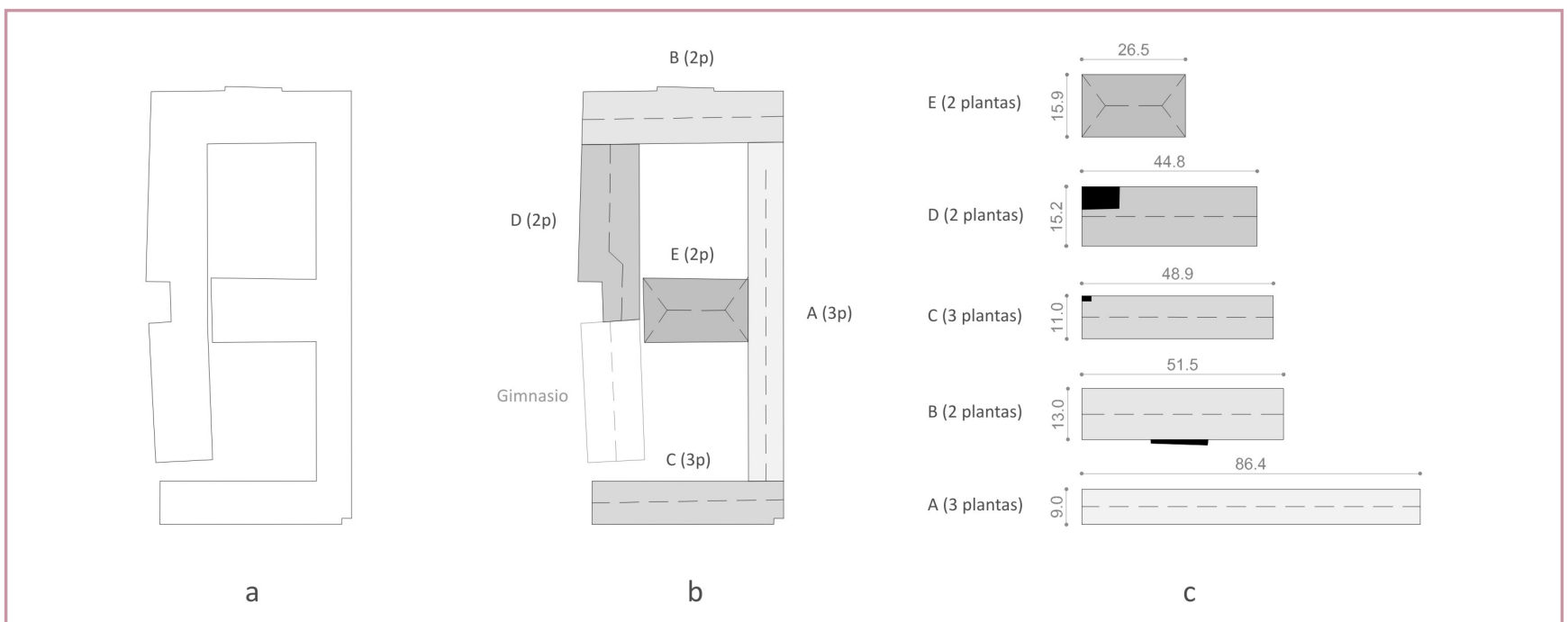

Figura 2. Metodología de caracterización formal de establecimientos educacionales (fuente: Elaboración propia).

urbana que presentan. Las universidades poseen la mayor extensión, en zonas concentradas en la modalidad de campus con un promedio de superficie de cubierta de $16.568 \mathrm{~m}^{2}$, formadas por agrupaciones de 10 o más edificios. Las escuelas, colegios y liceos, aparecen de forma dispersa ocupando superficies generalmente menores a una hectárea, compuestas por grupos de 1 a 5 edificios y con una superficie de cubierta promedio de $2.736 \mathrm{~m}^{2}$. Los jardines infantiles e institutos presentan también un patrón disperso, pero con menor

\section{ocupación territorial.}

\section{Caracterización formal y energética.}

Considerando los niveles formativos con mayor representatividad, se diferencian los establecimientos de nivel básico-medio -escuelas, colegios y liceos- de características similares y las universidades. Con el objetivo de reconocer las particularidades formales de los establecimientos educacionales, se desarrolla una caracterización formal en pabellones, presentada en la figura 2. Cada establecimiento definido por un polígono cerrado mudo (figura 2a) se diferencia en bloques según cambios de dirección en planta, altura de los volúmenes y programa (figura $2 \mathrm{~b}$ ), distinguiendo los bloques con predominancia de aulas, que son asimilados a rectángulos de dimensiones representativas (figura $2 \mathrm{c}$ ), a partir de los cuales se realiza un inventario de condiciones formales.

La caracterización de 15 establecimientos públicos de nivel básico-medio, con 57 bloques de aulas, permite comprobar largos representativos entre 30 y 40 m; anchos entre 9 y $11 \mathrm{~m}$ para crujías simples y entre 15 y 17 m para crujías dobles; igual distribución de número de plantas entre 1, 2 y 3 pisos; cubiertas mayoritariamente a 2 aguas y configuración espacial predominante en forma de C, pero que en todos los casos es asimilable a formas simples (lineales, en L o tipo patio). La misma metodología se utiliza para analizar el Campus Concepción de la Universidad del Bío-Bío, donde se reconocen 18 bloques con predominancia de aulas, con largos representativos también entre 30 y $40 \mathrm{~m}$, anchos de crujía entre 12 y $14 \mathrm{~m}$, cubiertas con formas variadas y configuración espacial predominante de bloques aislados.

A partir de datos de facturación de energía eléctrica, se analizan cinco establecimientos educacionales, comprobando que el consumo de energía eléctrica es de $11,0 \mathrm{kWh} / \mathrm{m}^{2}$-año en promedio para establecimientos de nivel básico-medio -normalmente sin climatización-y de 43,0 kWh/m²-año para nivel superior -con climatización parcial.

\section{SIMULACIÓN ENERGÉTICA CON SISTEMAS FOTOVOLTAICOS. Posición óptima de paneles fotovoltaicos para establecimientos educacionales.}

Con el objetivo de determinar la posición óptima de paneles fotovoltaicos para establecimientos educacionales en

Concepción, se realiza una evaluación multifactorial que considera la generación de energía eléctrica, el tiempo de autoconsumo -tiempo total de alimentación directa sin baterías- y los índices de compra y venta de energía, para paneles con diferente inclinación. La evaluación se realiza de acuerdo a un balance anual, asegurando una mayor distribución y contribución urbana de excedentes energéticos, los que se persiguen como aporte final de la investigación.

La caracterización formal realizada permite definir un modelo representativo de las condiciones encontradas. Lo anterior se lleva a cabo seleccionando un edificio de la Universidad del Bío-Bío - de 3 niveles y $306 \mathrm{~m}^{2}$ de superficie de cubierta-, para e que se establecen diferentes condiciones de simulación, definiendo casos de estudio representativos de los niveles básico-medio y superior.

La figura 3 muestra el modelo utilizado para realizar las simulaciones energéticas en Design Builder [v.4.2.0.054] con EnergyPlus 
NIVEL BÁSICO-MEDIO Escuela Rene Louvel Bert

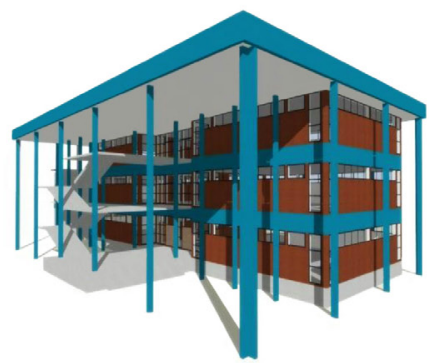

NIVEL SUPERIOR Aulario $A B$ - UBB
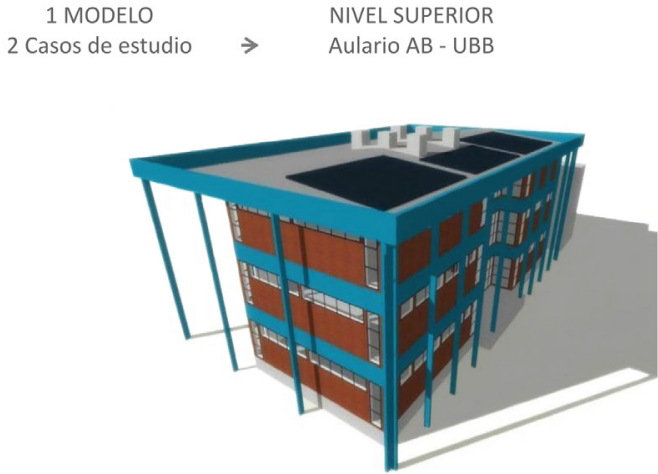

Figura 3. Modelo de simulación del edificio de Aulas AB de la Universidad del Bio-Bio (fuente: Elaboración propia).
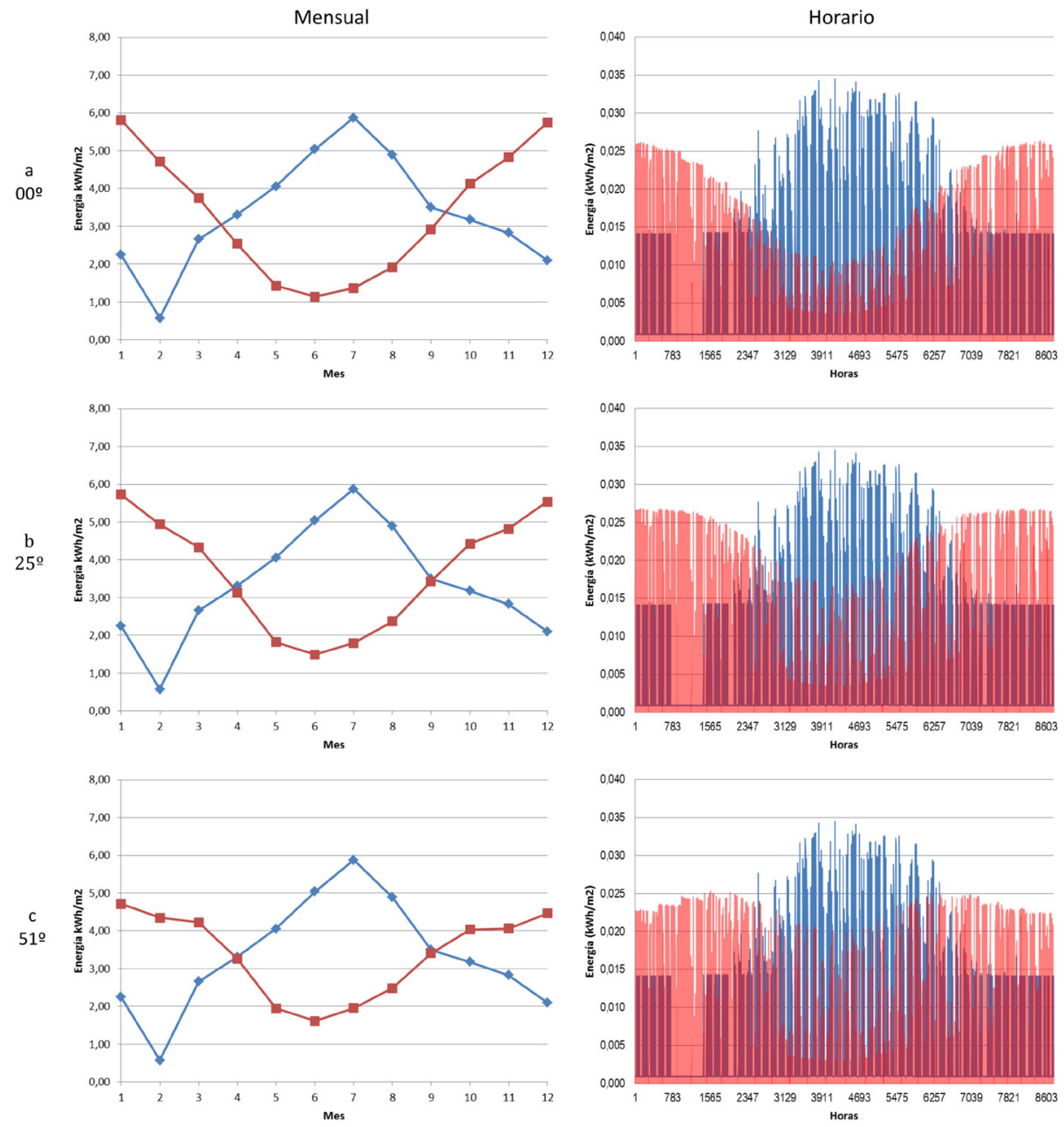

얼

[v.8.1] como motor de cálculo, utilizando el componente PV Generator - Simple, que permite un análisis de producción energética en etapas tempranas de diseño. Se considera un valor constante del 15\% de eficiencia, en correspondencia con la literatura internacional analizada (Tripathy et al. 2016).

Los resultados de simulación de la figura 4, considerando idéntica superficie de paneles fotovoltaicos con acimut norte y diferentes inclinaciones, permiten apreciar que la posición horizontal (figura 4a) produce excedentes de generación en los meses cálidos y déficit en los meses fríos, lo cual puede ser modificado al variar la inclinación de los paneles. La base de datos meteorológica utilizada (WMO Index number 856820_(WEC), permite comprobar la máxima radiación anual para acimut norte y $25^{\circ}$ de inclinación, y la máxima radiación para julio -mes de mayor consumo- para acimut norte y $51^{\circ}$ de inclinación. En base a esta información, se simulan las posiciones optimizadas para período anual (figura 4b) y para el mes de julio (figura 4c).

La tabla 1 muestra los resultados del análisis multifactorial para ambos niveles formativos asimilados a variaciones porcentuales que permite compararlos. Para todos los parámetros evaluados, la inclinación de $25^{\circ}$ obtiene el mejor desempeño, presentando mayor generación, igual o mayor tiempo de auto-consumo, más venta y menos compra de energía eléctrica. Esto demuestra la posición óptima de instalación de paneles fotovoltaicos para establecimientos educacionales en la comuna de Concepción. Los índices de compra y venta de energía permiten realizar un análisis de costo de ciclo de vida en base a la norma ASTM E 917-05, evaluando la relación entre la posición de sistemas fotovoltaicos de idéntica superficie y el tiempo de retorno de inversión (TRI). Considerando una tasa de descuento del 10\% (International Energy Agency 2010), un ciclo de vida de 40 años (National Renewable Energy Laboratory 2016) y una tasa de escalonamiento de electricidad del 6\% (Comisión Nacional de Energía, Ministerio de Energía, Gobierno de Chile), se comprueba que la posición optimizada presenta un TRI de 18 años; 4 años menor que la inclinación de $51^{\circ}$ y 5 años menor que la posición horizontal. Esto demuestra que pueden obtenerse menores TRI por la misma inversión, únicamente gracias a los mejores índices de compra y venta de energía eléctrica. 


\section{Aporte de energía solar en el desempeño energético.}

Se analiza la contribución de energía solar a los requerimientos energéticos de establecimientos educacionales, determinando la relación entre la superficie necesaria de captación y el consumo en distintos escenarios energéticos. El marco regulatorio energético chileno (contenido en el Reglamento de Ley No 20.5712), establece un sistema de incentivo para la generación en base a energías renovables, siendo la valorización de energía eléctrica comprada aproximadamente el doble de la vendida. Esta condición permite definir una ecuación de análisis donde:

Saldo de energía=Energía vendida/2-Energía comprada

Simulaciones realizadas con idéntica posición optimizada de paneles

fotovoltaicos -acimut norte e inclinación $25^{\circ}$ - y variando la superficie de captación, permiten comprobar una relación lineal entre la superficie de captación y el saldo de energía definido. Esta relación permite calcular la mínima superficie de capación que hace que el saldo de energía sea cero para distintos escenarios de demanda energética: Escenario A, cubriendo fuerza, iluminación y calefacción; Escenario B, cubriendo fuerza, iluminación con control de luminiscencia y calefacción; y Escenario C, cubriendo fuerza e iluminación con control de luminiscencia.

Los resultados de la figura 5 permiten comprobar el enorme potencial de aporte de la energía solar en el desempeño energético de los establecimientos educacionales, donde, en función de la geometría de cubierta, la contribución de energía solar permitiría cubrir todas las demandas energéticas (escenario A) de un establecimiento de nivel básico-medio de hasta 4 plantas o de nivel superior de hasta 3, con un saldo de energía, y por lo tanto de facturación anual, igual a cero. Pero asegurando, además, por la condición de saldo de energía establecida, que en todos

\section{SISTEMA DE DISEÑO PARAMÉTRICO DE OPTIMIZACIÓN SOLAR ACTIVA}

Las características formales y energéticas de establecimientos educacionales se combinan en una definición paramétrica, que permite sugerir decisiones de optimización volumétrica en etapas tempranas de diseño de nuevos establecimientos educacionales. Esto se lleva a cabo realizando una simulación de la forma del establecimiento, con la finalidad de determinar la capacidad solar activa de cubierta y sugerir volúmenes con mejores desempeños a partir de decisiones de implantación, configuración espacial y optimización de cubierta. La definición considera la instalación de sistemas fotovoltaicos paralelos a la cubierta, optimizando la forma de la misma en función de la posición de los sistemas. Si bien el alcance de esta investigación refiere a la optimización paramétrica para el uso de sistemas fotovoltaicos, es importante tener en cuenta que el análisis ambiental en etapas tempranas de diseño debe considerar muchos otros requerimientos funcionales y energéticos que escapan de los objetivos de este trabajo, pero que bien podrían incorporarse en etapas sucesivas.

$$
\text { - Escenario A Escenario B Escenario C }
$$

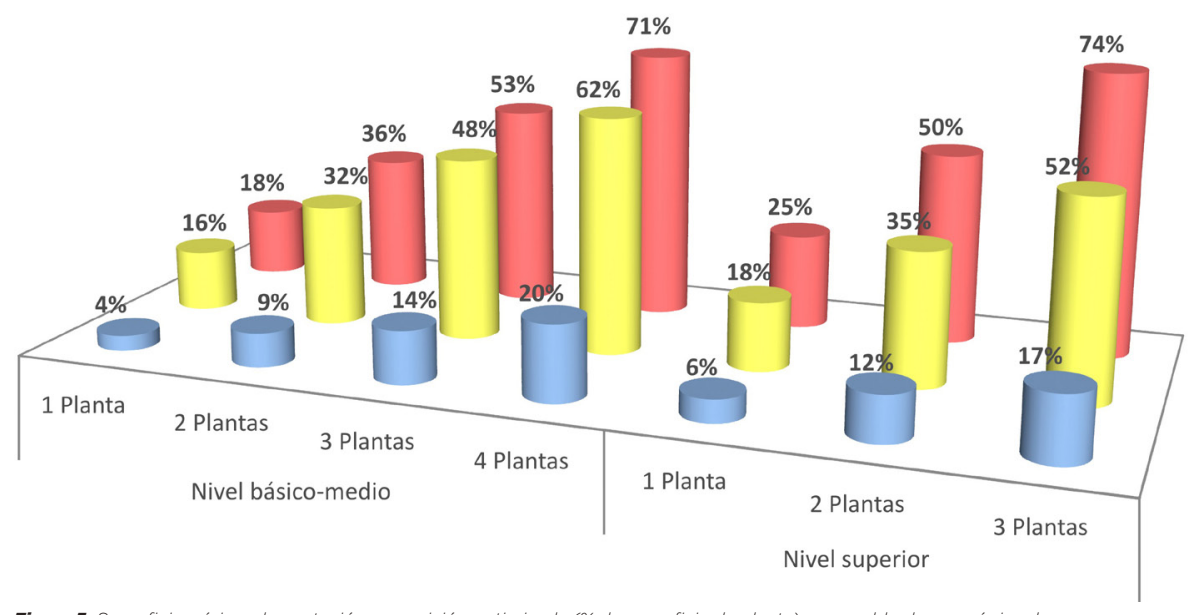

Figura 5. Superficie mínima de captación en posición optimizada (\% de superficie de planta), para saldo de energía igual a cero y según distintos escenarios, cantidad de plantas y nivel formativo (fuente: Elaboración propia).

\begin{tabular}{|c|c|c|c|c|c|c|c|c|c|}
\hline \multirow[t]{2}{*}{ NIVEL } & \multirow{2}{*}{$\begin{array}{c}\text { INCLINACIÓN } \\
\qquad-\end{array}$} & \multicolumn{2}{|c|}{$\begin{array}{l}\text { GENERACIÓN } \\
\text { DE ENERGÍA }\end{array}$} & \multicolumn{2}{|c|}{$\begin{array}{c}\text { TIEMPO DE } \\
\text { AUTO-CONSUMO } \\
\end{array}$} & \multicolumn{2}{|c|}{ VENTA DE ENERGÍA } & \multicolumn{2}{|c|}{ COMPRA DE ENERGIA } \\
\hline & & $\mathrm{kWh} / \mathrm{m}^{2}$-año & $\%$ & Horas & $\%$ & $\mathrm{kWh} / \mathrm{m}^{2}$-año & $\%$ & $\mathrm{kWh} / \mathrm{m}^{2}$-año & $\%$ \\
\hline \multirow{3}{*}{ Superior } & 00 & 40,29 & 0,0 & 2.590 & 0,0 & 25,67 & 0,0 & 25,65 & 0,0 \\
\hline & 25 & 43,82 & 8,8 & 2.665 & 0,9 & 28,38 & 10,5 & 24,83 & $-3,2$ \\
\hline & 51 & 40,52 & 0,6 & 2.573 & $-0,2$ & 25,55 & $-0,5$ & 25,31 & $-1,3$ \\
\hline \multirow{3}{*}{ Básico-medio } & 00 & 10,61 & 0,0 & 3.612 & 0,0 & 8,30 & 0,0 & 8,32 & 0,0 \\
\hline & 25 & 11,58 & 9,2 & 3.612 & 0,0 & 8,94 & 7,7 & 7,98 & $-4,0$ \\
\hline & 51 & 10,79 & 1,7 & 3.596 & $-0,2$ & 8,15 & $-1,8$ & 7,99 & $-3,9$ \\
\hline
\end{tabular}

Tabla 1. Comparativo multifactorial según inclinación de paneles fotovoltaicos (fuente: Elaboración propia). 


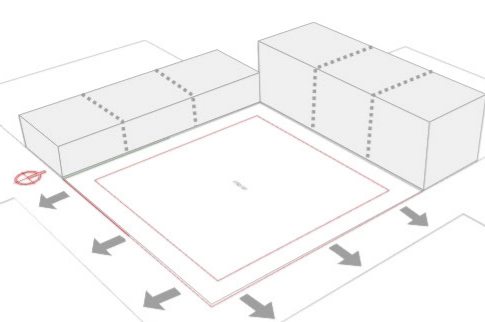

A - Definición de contexto

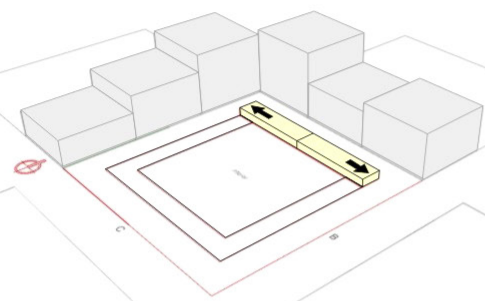

D - Etapa $1_{\text {_ Lineal al norte }}$

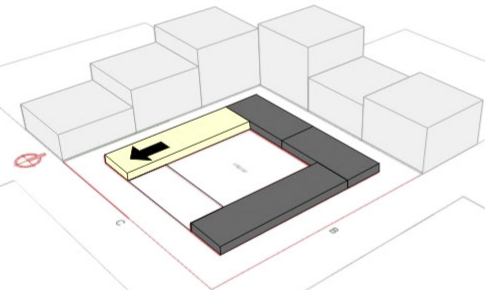

G - Etapa 3 _ Tipo C al oeste

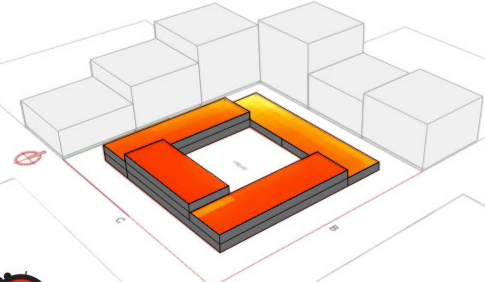

Ladybug!

J - Evaluación de radiación en cubierta

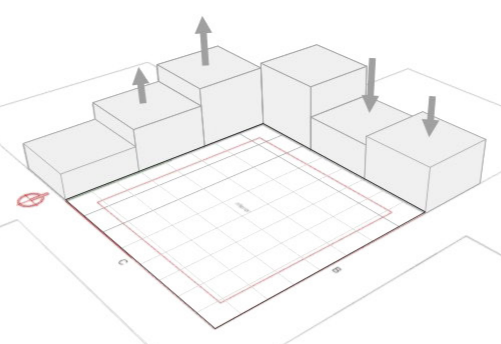

B - Definición de perfil urbano

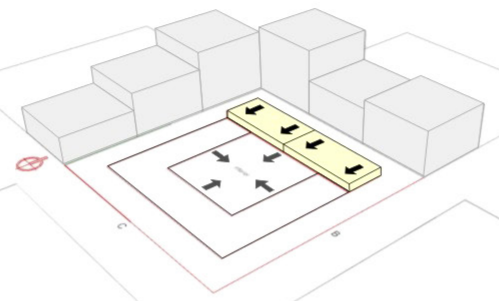

E - Ajuste ancho de crujía

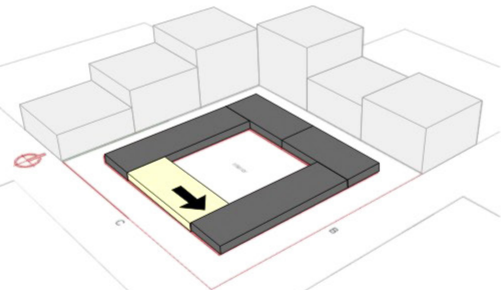

H - Etapa 4 _ Tipo patio al sur

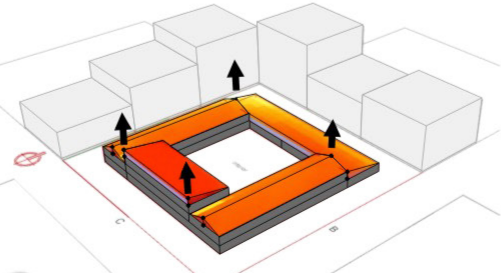

Galapagos!

K - Optimización formal de cubierta

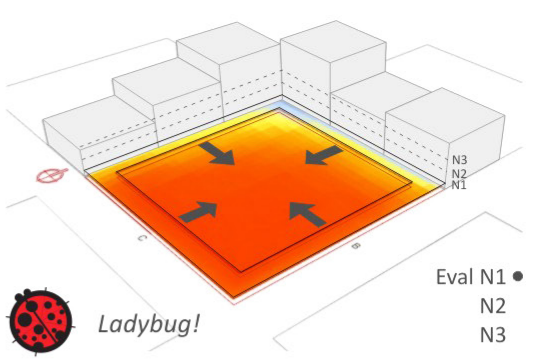

C - Evaluación de radiación y deslinde

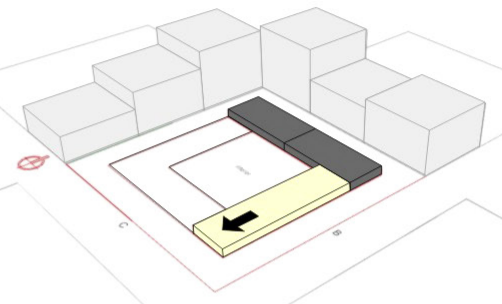

F - Etapa 2 _ Tipo L al este

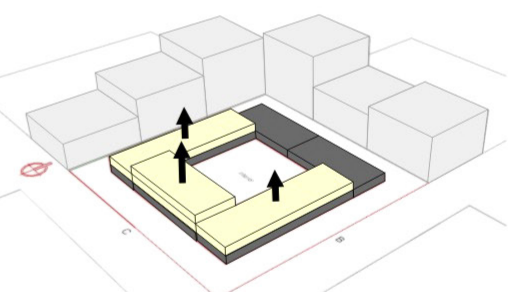

I - Definición de número de plantas

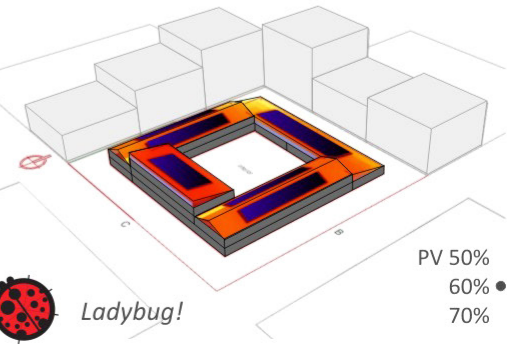

L - Definición de sistemas fotovoltaicos

Figura 6. Entorno visual y secuencia de definición paramétrica de optimización solar activa (fuente: Elaboración propia). 


\section{Definición paramétrica de optimización solar activa.}

Se realiza una definición paramétrica en Grasshopper (v.0.9.0076), utilizando el plugin de análisis ambiental Ladybug (v.0.0.62) (Sadeghipour y Pak 2013) -con base de archivos climáticos EnergyPluspara análisis de radiación solar y cálculo de sistemas fotovoltaicos, optimizando los resultados con el módulo de soluciones evolutivas y algoritmos genéticos Galapagos (Rutten 2010)

La configuración espacial se programa mediante un patrón de crecimiento establecido a partir de antecedentes (Agencia Chilena de Eficiencia Energética 2012), y expresa la influencia de la orientación en la demanda energética, considerando, en primer lugar la orientación norte (10,5 kWh $/ \mathrm{m}^{2}$-año) mediante un bloque lineal; seguido por la orientación este (15,0 kWh/m²-año), que define una configuración en L; la oeste (16,0 kWh/ $\mathrm{m}^{2}$-año), definiendo una configuración en C; y la orientación sur, (20,0 kWh/m²año), que consolida la configuración tipo patio. Por otra parte, se establecen reglas paramétricas de diseño formal de cubierta, considerando, por defecto, la incorporación de paneles fotovoltaicos con posición optimizada, pero permitiendo variaciones formales y distintas configuraciones. La figura 6 muestra el entorno visual y la secuencia de definición paramétrica desde una forma lineal hasta un edificio con patio central, que permite configurar interactivamente el diseño volumétrico del establecimiento y su contexto, evaluando en tiempo real el potencial solar activo mediante Ladybug (Ladybug_Radiation Analysis y Ladybug_Photovoltaics Surface) y optimizando el diseño de cubierta mediante Galapagos. La definición permite especificar características formales, energéticas y económicas para calcular la radiación solar recibida, producción energética, porcentaje cubierto de consumo, tamaño de la instalación fotovoltaica y costo estimativo de inversión.

\section{Ensayo exploratorio de formas con mayor potencial de generación fotovoltaica.}

Con el objetivo de ejemplificar el potencial de la definición paramétrica desarrollada, se presenta un ensayo exploratorio de formas con mayor potencial de generación fotovoltaica, considerando una eficiencia de módulos del $15 \%$ y el consumo real determinado en la caracterización energética, que incluye iluminación y equipos en nivel básico-medio, y sumando calefacción en nivel superior.

La figura 7 muestra el modelo de simulación, considerando una ubicación en la trama central de Concepción, superficie de cubierta de $2.500 \mathrm{~m}^{2}$-representativa del promedio de nivel básico-medio, ocupación del $70 \%$ de la superficie de cubierta de captación- en función de las restricciones de operación y servicio, y bloques de 9 m de ancho de crujía para maximizar la superficie de ocupación de paneles fotovoltaicos dispuestos en posición optimizada. La simulación considera ambos niveles formativos y variaciones progresivas en el número de plantas de cada bloque, evitando auto-sombreados. Esto permite evaluar el porcentaje de consumo

cubierto y los excedentes energéticos, que son comparados con el consumo eléctrico promedio de una vivienda en Concepción, de superficie promedio de $77 \mathrm{~m}^{2}$ y un consumo de electricidad de $31,5 \mathrm{kWh} / \mathrm{m}^{2}$-año destinados a equipos e iluminación (Corporación de Desarrollo Tecnológico 2010)

Los resultados de la tabla 2 muestran que en el nivel básico-medio, para todas las configuraciones de número de plantas por bloque, la generación de energía permitiría cubrir el consumo del establecimiento, con excedentes para cubrir el consumo eléctrico total anual de hasta 60 viviendas. Mientras que en el nivel superior, dependiendo de la configuración, podrían producirse remanentes para cubrir el consumo electico total anual de hasta 33 viviendas.

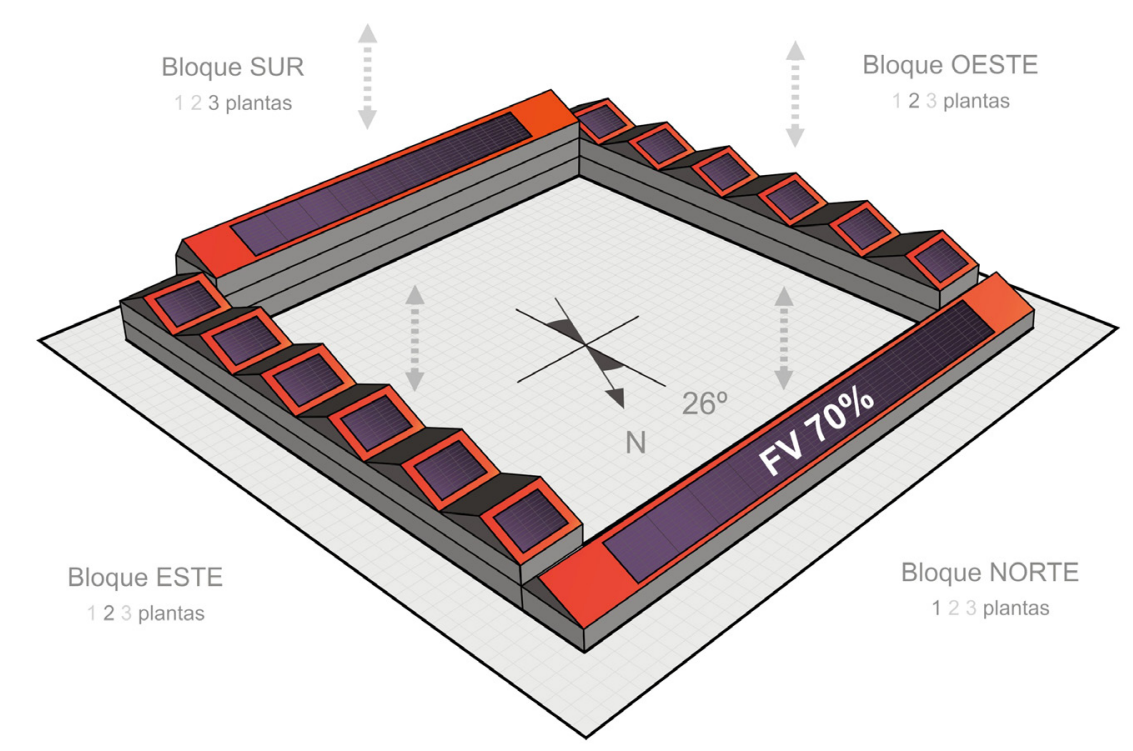

Figura 7. Modelo de simulación en ensayo de potencial de generación energética (fuente: Elaboración propia). 


\begin{tabular}{|c|c|c|c|c|c|}
\hline \multirow{2}{*}{$\begin{array}{c}\text { NIVEL } \\
\text { FORMATIVO }\end{array}$} & \multicolumn{4}{|c|}{ NÚMERO DE PLANTAS POR BLOQUE } & \multirow{2}{*}{$\begin{array}{c}\text { CONSUMO CUBIERTO } \\
\%\end{array}$} \\
\hline & Norte & Este & Oeste & Sur & \\
\hline \multirow{7}{*}{$\begin{array}{l}\text { Básico } \\
\text { Medio }\end{array}$} & 1 & 1 & 1 & 1 & $749 \%$ \\
\hline & 1 & 1 & 1 & 2 & $621 \%$ \\
\hline & 1 & 2 & 2 & 2 & $440 \%$ \\
\hline & 2 & 2 & 2 & 2 & $379 \%$ \\
\hline & 2 & 2 & 2 & 3 & $341 \%$ \\
\hline & 2 & 3 & 3 & 3 & $278 \%$ \\
\hline & 3 & 3 & 3 & 3 & $252 \%$ \\
\hline \multirow{7}{*}{ Superior } & 1 & 1 & 1 & 1 & $194 \%$ \\
\hline & 1 & 1 & 1 & 2 & $159 \%$ \\
\hline & 1 & 2 & 2 & 2 & $113 \%$ \\
\hline & 2 & 2 & 2 & 2 & $97 \%$ \\
\hline & 2 & 2 & 2 & 3 & $87 \%$ \\
\hline & 2 & 3 & 3 & 3 & $71 \%$ \\
\hline & 3 & 3 & 3 & 3 & $65 \%$ \\
\hline
\end{tabular}

\begin{tabular}{|c|}
\hline EXCEDENTE DE ENERGÍA \\
\hline $\mathrm{kWh} / \mathrm{m}^{2}$-año \\
\hline 146.534 \\
\hline 141.976 \\
\hline 131.279 \\
\hline 125.466 \\
\hline 120.716 \\
\hline 109.715 \\
\hline 103.238 \\
\hline 80.870 \\
\hline 62.488 \\
\hline 18.959 \\
\hline 0 \\
\hline 0
\end{tabular}

\begin{tabular}{|c|}
\hline NÚMERO DE VIVIENDAS \\
\hline u \\
\hline 60 \\
\hline 59 \\
\hline 54 \\
\hline 52 \\
\hline 50 \\
\hline 45 \\
\hline 43 \\
\hline 33 \\
\hline 26 \\
\hline 8 \\
\hline 0
\end{tabular}

Tabla 2. Consumo cubierto
(fuente: Elaboración propia).

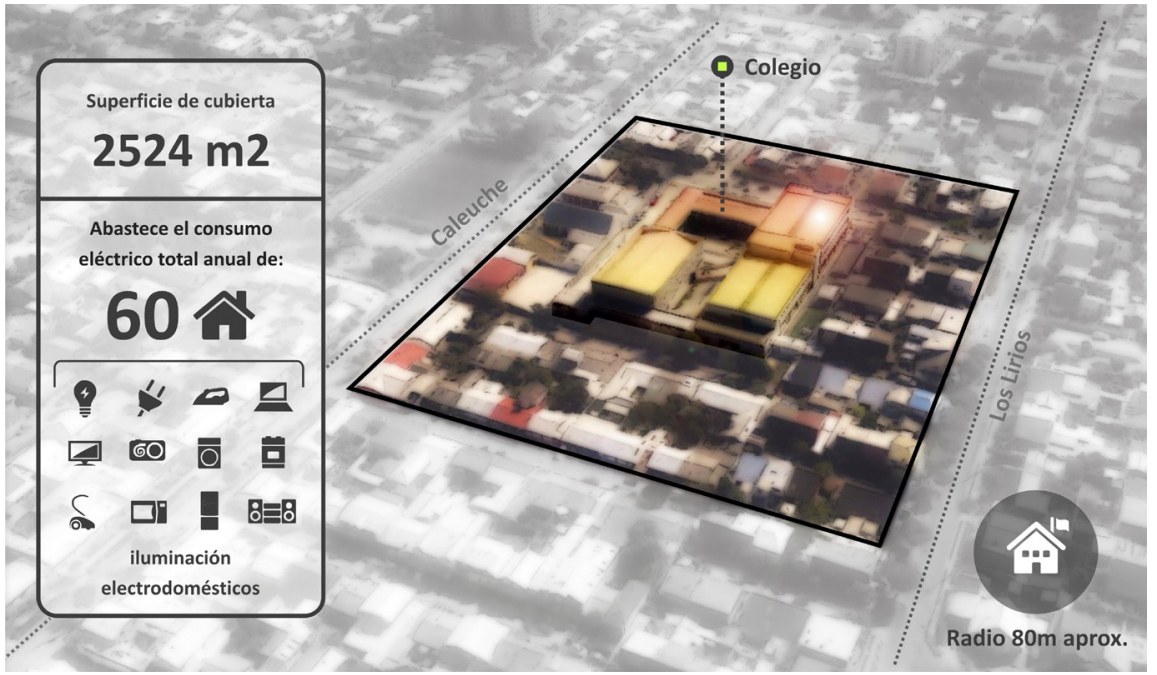

Figura 8. Imagen objetivo de potencial de abastecimiento eléctrico urbano de un establecimiento de superficie promedio (fuente:
La figura 8 muestra una imagen objetivo del potencial de abastecimiento urbano de energía eléctrica de un establecimiento existente de superficie de cubierta similar al evaluado en el ensayo exploratorio, que podría abastecer el consumo eléctrico total anual de cerca de 60 viviendas, cuando la configuración del número de plantas por bloque es uniforme de 1 planta. Se trata de una estimación primaria -sin considerar densidad ni morfología urbana- pero que permite tener un acercamiento al aporte energético urbano que podría lograrse mediante la integración de sistemas fotovoltaicos debidamente orientados en establecimientos educacionales para maximizar sus capacidades. 


\section{CONCLUSIONES Y FUTURAS LÍNEAS DE INVESTIGACIÓN.}

Este trabajo demuestra que, por su extensión volumétrica, los establecimientos educacionales presentan un gran potencial de captación activa de energía solar, incluso en ciudades de latitudes medias como Concepción, Chile. Además, esta posibilidad puede optimizarse mediante estrategias de diseño de la forma arquitectónica. En países en desarrollo, esta optimización se justifica especialmente por el elevado costo de las instalaciones solares, lo que representa una barrera para su implementación. Los resultados demuestran que el potencial solar disponible permite generar establecimientos con autosuficiencia eléctrica y con remanentes para mejorar el confort, activar sus espacios para fines sociales en períodos sin uso, compartir localmente o aportar a sistemas inteligentes de distribución de electricidad (smart grid), basados en su dispersión territorial. Esta visión de los establecimientos educacionales como edificios de energía positiva, invita a reflexionar sobre su contribución en futuros escenarios energéticos, en los que podrían consolidarse como nodos urbanos de generación energética distribuida. Así, cumplirían con un importante papel en el desarrollo de comunidades urbanas sustentables y sintonizarían con una nueva generación -energética y humana- donde el edificio actúe como educador en prácticas sostenibles, hacia nuevos modelos de democracia y descentralización energética. Se ha demostrado que la definición paramétrica constituye una herramienta eficaz en la optimización de la forma arquitectónica de establecimientos educacionales para su mayor potencial solar. Se ha logrado construir un procedimiento básico para incorporar la dimensión solar activa en el diseño temprano de infraestructuras educacionales, que, gracias al carácter de código abierto, podría ampliarse integrando también otros aspectos, como el factor económico, la conformación urbana o el beneficio social de los excedentes energéticos.

\section{REFERENCIAS}

Agencia Chilena de Eficiencia Energética (ACHEE), 2012. Guía de Eficiencia Energética Para Establecimientos Educacionales (GEEEduc). Santiago de Chile: Agencia Chilena de Eficiencia Energética. Disponible en: https:// www.acee.cl/biblioteca/

Anton. I. y Tănase, D. 2016. "Informed Geometries. Parametric Modelling and Energy Analysis in Early Stages of Design." Energy Procedia, 85, 9-16.

Comisión Nacional de Energia, Ministerio de Energía, Gobierno de Chile, - http://energiaabierta.cl Corporación de Desarrollo Tecnológico, 2010. Estudio de Usos Finales y Curva de Oferta de Conservación de la Energía en el Sector Residencial.

Depecker, P., Menezo, C., Virgone, J. y Lepers, S., 2001. "Design of Buildings Shape and Energetic Consumption." Building and Environment, 36, (5): 627-35.

García, R. y González, A., 2014. "Condiciones de Forma Y Desempeño Energético de Viviendas Unifamiliares En El Centro Sur de Chile." Revista INVI, 29, (80): 111-141.

Garcia, R., Wegertseder, P., Baeriswyl, S. y Trebilcock, M., 2014. "Mapa Energético-Solar de Concepción : Cartografía Urbana Del Consumo Energético Y Captación Solar En Edificaciones Residenciales de Concepción. Chile." Norte Grande, 59, 123-144.

Hachem, C., Athienitis, A. y Fazio, P., 2011. "Parametric Investigation of Geometric Form Effects on Solar Potential of Housing Units." So/ar Energy, 85, (9): 1864-1877.

Instituto Nacional de Estadistica de Chile (INE), 2002. Censo de Población y Vivienda 2002. Disponible en: https://redatam-ine.ine.cl/redbin/RpWebEngine.exe/Portal?BASE=CENSO 2002\&lang=esp

International Energy Agency, 2010. Projected Costs of Generating Electricity.

Lund, P. 2012. "Large-Scale Urban Renewable Electricity Schemes - Integration and Interfacing Aspects." Energy Conversion and Management, 63, 162-72.

Ministerio de Educación, Gobierno de Chile, (s/f). Plan Estratégico de Infraestructura Escolar. Ministerio de Energía, Gobierno de Chile, 2014a. Energía 2050: Politica energética de Chile.

Ministerio de Energia, Gobierno de Chile, 2014b. Energías renovables en Chile.
Ministerio de Energia Gobierno de Chile, 2014c Reglamento de Ley No 20.571 - Regula El Pago de Las Tarifas Eléctricas de Las Generadoras Residenciales. Santiago de Chile: Diario Oficial de la República de Chile Disponible en: https://www.leychile.cl/Navegar?idNorma=1066257

Naciones Unidas, 2014. World Urbanization Prospects. Demographic Research (Vol. 12).

National Renewable Energy Laboratory, 2016. Distributed Generation Energy Technology Operations and Maintenance Costs.

Olgyay, V., 1962. Design with Climate - Bioclimatic Approach to Architectural Regionalism. Nueva Jersey Princeton University.

Rutten, D., 2010. Evolutionary Principles Applied to Problem Solving. Disponible en: http://www. grasshopper3d.com/profiles/blogs/evolutionary-principles.

Sadeghipour, M. y Pak, M., 2013. "Ladybug: A Parametric Environmental Plugin for Grasshoper To Help Designers Create an Environmentally-Conscious Design." En 13th Conference of International Building Performance Simulation Association, 3129-3135. Disponible en: http://www.ibpsa.org/proceedings/ bs2013/p_2499.pdf.

Salcedo. P. 2012 "Análisis Paramétrico de Volúmenes Arquitectónicos Con Algoritmos Genéticos." Hábitat Sustentable, 2, 47-58.

Tripathy, M., Sadhu, P. y Panda, S., 2016. "A Critical Review on Building Integrated Photovoltaic Products and Their Applications." Renewable and Sustainable Energy Reviews, 61, 451-65.

Troncoso, L, Garcia, R. Nope. A. y Arellano, R., 2015. "Mansardas Solares Por Optimización Paramétrica Y Fabricación Digital Para Viviendas NZE." Blucher Design Proceedings, 2, (3): $317-321$. Tuhus-Dubrow, D. y Krarti, M., 2010. "Genetic-Algorithm Based Approach to Optimize Building Envelope Design for Residential Buildings." Building and Environment, 45, (7). 1574-158.

Zalamea, E. y García, R., 2014. "Roof Characteristics for Integrated Solar Collection in Dwellings of Real- Estate Developments in Concepción, Chile." Revista de La Construcción Journal of Construction, 36, (133): 36-44. 